\title{
A new tool for measuring the suckling stimulus during breastfeeding in humans: the orokinetogram and the Fourier series
}

\author{
L. M. Voloschin ${ }^{1,2 *}$, O. Althabe ${ }^{1}$, H. Olivé ${ }^{1}$, V. Diena ${ }^{1}$ and B. Repezza ${ }^{1}$ \\ ${ }^{1}$ Laboratorio de Investigaciones en Neuroendocrinología de la Reproducción Humana (LINERH), \\ Hospital General de Agudos Dr Carlos G. Durand, Ambroseti 743 CP 1405, Buenos Aires, Argentina; \\ and 'Instituto de Neurobiología, Serrano 669 CP 1414, Buenos Aires, Argentina
}

\begin{abstract}
The Fourier series was used to analyse the oral movements recorded by the orokinetogram during breastfeeding in human babies. This is a new method that allows recording of oral movements without introducing any extrinsic element between the nipple and the mouth of the baby. The advantage of displaying suckling activity after fast Fourier transform (FFT) is that this algorithm allows storage, quantification and frequency analysis of the oral movements throughout a suckling bout, which enables the total oral activity to be measured. Two types of oral movements are found: slow high amplitude (SHA) and fast low amplitude (FLA). FLA movements may be derived from peristaltic movements of the tongue that result in tickling stimuli to the mechanoreceptors of the nipple and milk expression. The frequency bandwidth of oral movements is wider $(0-8 \mathrm{~Hz})$ than has been described previously $(0-3 \mathrm{~Hz})$ and this is due to the presence of the FLA oral movements. An indirect measurement of the energy of oral movements during suckling is obtained by the pattern of energy distribution used in each individual frequency band by oral movements. This pattern changes in relation to the periods of continuous and intermittent suckling activity. SHA and FLA oral movements are more intense during continuous suckling. Statistical analysis showed a correlation between the energy of SHA and FLA waves throughout the suckling bout, and also that the highest level of energy during suckling activity is displayed during the first $2 \mathrm{~min}$. The novel tools described in this paper allow investigation of the role of suckling stimulus in reflex hormone release and other mother-infant interactions.
\end{abstract}

\section{Introduction}

During breastfeeding, a baby obtains nourishment and at the same time induces reflex hormonal changes in the mother which bring about milk production and postpartum amenorrhoea (Howie et al., 1982; Díaz et al., 1988; Yokoyama et al., 1994). The stimulus of suckling is composed of a series of oral movements that both draw the milk out of the lactiferous sinuses and stimulate nipple mechanoreceptors, which in turn trigger reflexes resulting in the release or inhibition of hormones in the mother.

The oral movements of babies during suckling have been thoroughly investigated (Wolff, 1968; Dreweet and Wooldridge, 1979). However, to date, only partial measurements of the magnitude of suckling movements have been determined because the amplitude and frequency of oral movements have not been quantified simultaneously throughout an entire suckling bout. It is important to determine the overall magnitude of this stimulus to explore the reflex action of suckling during breastfeeding and it is

*Correspondence address: Instituto de Neurobiologia, Serrano 669 CP 1414, Buenos Aires, Argentina.

Revised manuscript received 30 June 1998. necessary to consider three dimensions: amplitude, frequency and period of suckling. It is possible to quantify and analyse the oral movements throughout the entire suckling bout by means of the Fourier series. The advantage of displaying suckling activity in the frequency domain after FFT is that this algorithm allows storage, quantification and frequency analysis of all the periodical waveforms produced by oral movements throughout a suckling bout, enabling measurement of the total oral activity. Therefore, it is possible to quantify and statistically analyse the overall magnitude of the suckling stimulus in breastfed babies. In this study, the oral movements of breastfed babies during suckling bouts were recorded using the orokinetogram, a novel method that allows recording of high and low amplitude movements without introducing any extrinsic element between the nipple and the baby's mouth.

\section{Materials and Methods}

\section{Subjects}

A total of 30 healthy breastfed babies aged between 1 and 2 months with a fasting period of no less than $3 \mathrm{~h}$ were 
studied. The babies were divided into two groups. Pressure transducer and orokinetogram recordings were taken simultaneously from one group of ten babies. In the second group of 20 babies, only the orokinetogram was used. The data of the waveforms produced by oral movements were statistically analysed in six babies from the second group during a 4-6 min suckling bout without arrest. Oral movements during the suckling bout were recorded from the moment the infants were attached to the nipple until their spontaneous detachment. Infants were correctly positioned and attached to the breast during breastfeeding. Babies were weighed before and after the suckling period with an electronic digital balance to an accuracy of $0.1 \mathrm{~g}$. A few babies were excluded from the study because their body weight did not increase after the suckling period. These babies did not readily attach to the nipple and had short periods of suckling. It is possible that these babies had not been fasted for the specified $3 \mathrm{~h}$.

Mothers were aged between 20 and 25 years with normal nutrition. The research protocol was approved by the Hospital General de Agudos Dr Carlos G. Durand ethical committee. All the mothers received appropriate information and signed a consent form before entering the study.

\section{Orokinetogram}

Two recording electrodes were placed on each infant: the first was attached to the chin near the lower lip on the skin covering the bone of the lower jaw; the second was placed between the first electrode and the hyoid bone on the skin covering the muscles of the tongue. A ground electrode was placed on the baby's forehead. Electrodes were held in place by means of sparadrape tape (Micropore 3M). Oral movements produced variations in the electrical resistance of the skin between the two recording electrodes, which was amplified and recorded (Akonic Polygraph, Argentina).

\section{Intra-oral pressure}

A Silastic catheter (Dow Corning, Midland, MI) was filled with sterile water and fixed by means of sparadrape tape to the external side of the breast so that its free end was at the level of the nipple. The free end of the catheter was closed so that it recorded only positive pressure exerted on the nipple during suckling activity. During suckling, the babies held the nipple and the catheter together. The other end of the catheter was connected to a Statham PB-23 pressure transducer, and the signals obtained were amplified.

\section{Orokinetogram and pressure transducer recordings}

Simultaneous orokinetogram and pressure transducer recordings were obtained to enable comparisons to be made.

\section{Digitalization and analysis of the signals}

Signals obtained by the orokinetogram and pressure transducer recordings were digitalized by means of a Keithel
DAS 1601 analog/digital I/O board. Signals were recorded, analysed and stored magnetically. The FFT algorithms were used to analyse the periodical wave signals produced by oral movements during suckling. Oral movements were analysed in frames of $30 \mathrm{~s}$ of suckling. When a pressure transducer records high and low intensity signals, the high intensity signals may saturate because of the high gain used to detect the low intensity signals. The orokinetogram simultaneously recorded high and low amplitude signals without saturation. These recordings were used to analyse the oral movements because saturated signals are unsuitable for analysis with the Fourier series.

\section{The three-dimensional pattern of energy in terms of frequency of oral movements during suckling}

The autopower spectral density (APSD) function was used to analyse in three dimensions the amplitude and frequency of oral movements and their variations throughout the suckling bout. The APSD function uses the signals after autocorrelation and calculates the normalized power of each individual frequency channel from the amplitude spectrum that is output by the FFT algorithm. It shows the power in each frequency band and represents the distribution of energy in terms of frequency (Ramirez, 1985). The power of each frequency channel is proportional to the amplitude and to the number of the periodical oral movements that matches the frequency channel. Therefore, the APSD is an indirect measurement of the energy used by the oral movements in each one of the different frequencies displayed. The APSD unit is $\mathrm{V}^{2} \mathrm{~Hz}$.

\section{Statistical analysis}

The following measurements were determined in a group of six babies that were fed during a $4-6$ min suckling bout, without detachment from the nipple.

Total amount of power displayed in the distribution of energy in terms of frequency throughout the suckling bout. The area under the curve of the APSD represents the total amount of power displayed in the distribution of energy in terms of frequency. The area under the curve of each consecutive APSD frame of $30 \mathrm{~s}$ throughout the whole suckling period was calculated to determine the amount of energy displayed in each frame. The amount of the energy was measured in arbitrary units. The maximum energy was considered to be $100 \%$, and the energies of other frames were calculated as a proportion of the maximum throughout the suckling bout. Means \pm SEM from the area under the curve of each one of the successive APSD frames of all babies were calculated. The presence of linear component trends in the data was checked by means of tests for the homogeneity of individual longitudinal regression lines, accounting for intra- and intersubject variability (Crowder and Hand, 1990).

Temporal relationships between slow and fast oral movements throughout the suckling bout. In each frame of $30 \mathrm{~s}$, averages 


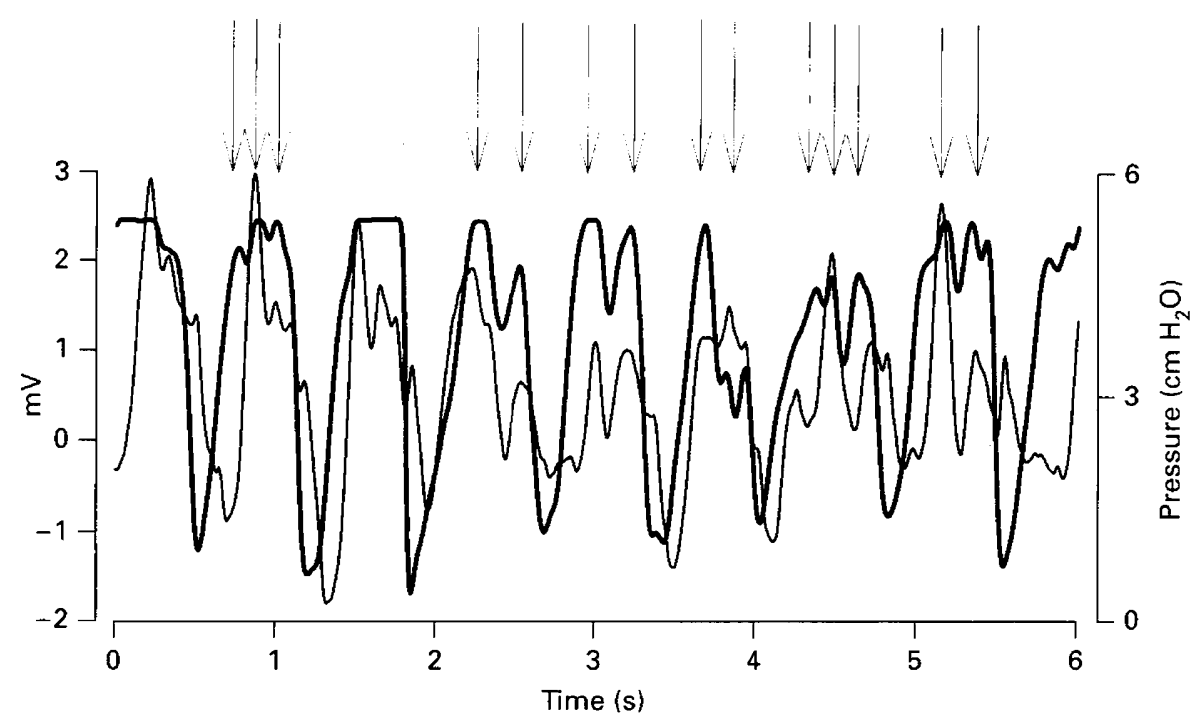

Fig. 1. Simultaneous recording of oral movements of breastfeeding human babies using a pressure transducer (-) and an orokinetogram (-). Arrows show fast low amplitude oral movements that are superimposed on slow high amplitude oral movements. These two types of oral movement are concurrently displayed by the orokinetogram and by the transducer which detects positive pressure at the level of the nipple.

of APSD data were separately calculated for SHA and FLA frequency channels. Statistical regression and correlation between FLA and SHA averages throughout the entire suckling bout were calculated for each baby.

\section{Results}

Simultaneous recordings of oral movements obtained by the orokinetogram and by the pressure transducer at the level of the nipple were similar (Fig. 1). SHA and FLA oral movements were observed using both methods; the FLA movements were mainly superimposed on the SHA movements. When the orokinetogram was used, recordings of both SHA and FLA oral movements were easily detected, whereas with the pressure transducer, FLA oral movements could only be detected after a high gain, which can cause saturation of the signal. With both recording systems, the display of SHA and FLA movements was consistent within infants provided that the signals from the pressure transducer did not saturate. When this happened, only SHA movements were consistently observed. Continuous and intermittent periods of suckling activity were observed with both recording methods.

The waveforms from the oral movements can be displayed in time (Fig. 2a) and frequency domain (Fig. 2b). Oral movements displayed in frequency after FFT or time domains are indistinct, as once the FFT is transformed back to the time domain by the inverse fast Fourier transform (IFFT) algorithm (Fig. 2c), the result is similar to that displayed by the time domain.

Continuous and intermittent periods of suckling activity can be displayed in time and frequency domains (Fig. 3a,b). The orokinetogram traces and the distribution of energy in terms of frequency changes throughout the suckling bout in relation to the periods of continuous or intermittent suckling. SHA oral movements under $1 \mathrm{~Hz}$ predominate during continuous suckling and shift to a higher frequency during

(a)

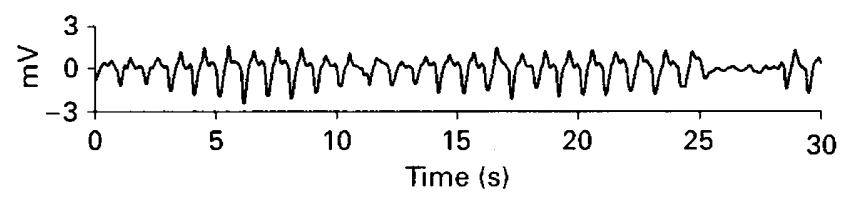

(b)

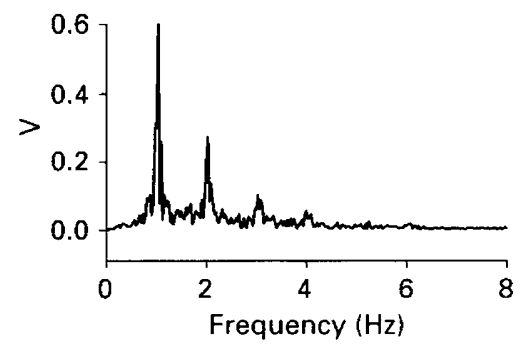

(c)

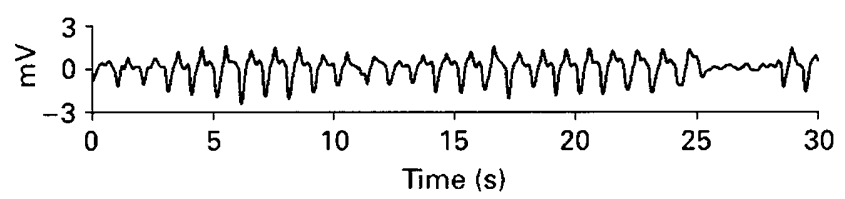

Fig. 2. The waveforms from oral movements of breastfeeding human babies displayed in (a) time domain, (b) frequency domain after fast Fourier transform (FFT), and (c) back to time domain by inverse fast Fourier transform. Once FFT is transformed back to the time domain by the inverse FFT algorithm, the result is similar to that for the time domain (a). 
(a)
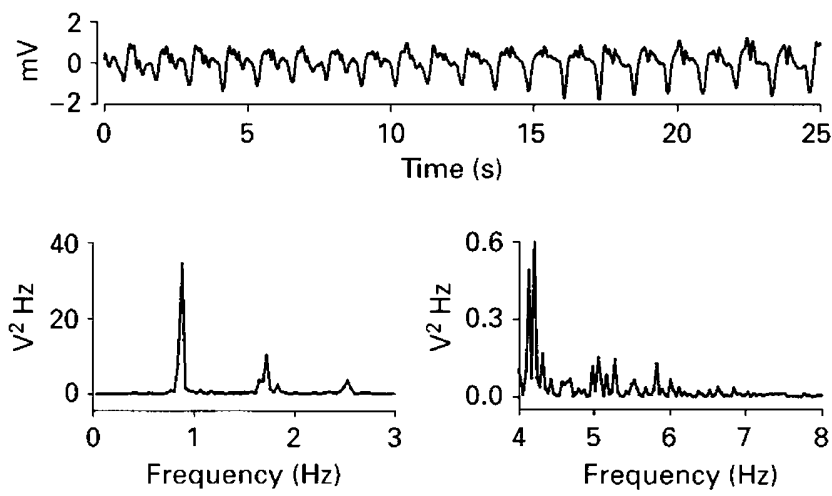

(b)
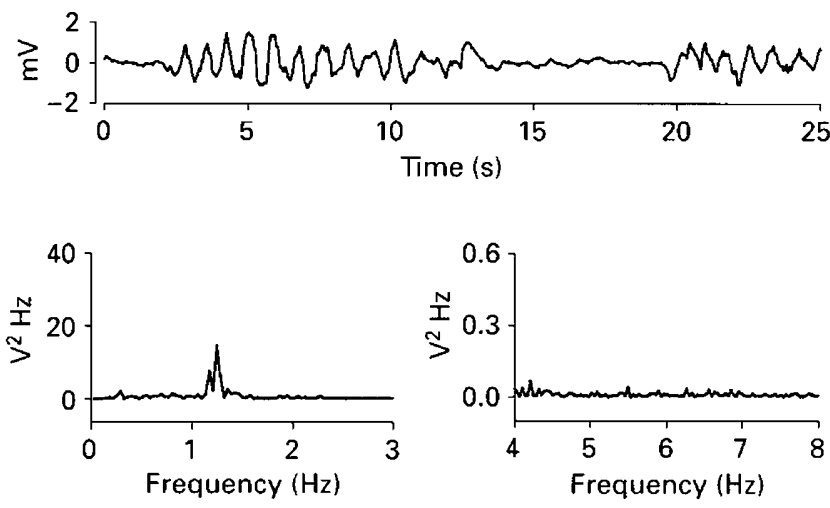

Fig. 3. Samples (25s) of (a) continuous and (b) intermittent periods of suckling activity in breastfed human babies. In each sample, the oral movements are displayed in time (upper trace) and in frequency domain at which slow high amplitude (SHA) (left lower trace) and fast low amplitude (FHA) (right lower trace) channels are shown. SHA and FLA movements are more intense during continuous suckling. SHA movements under $1 \mathrm{~Hz}$ predominate during continuous suckling. During intermittent suckling, movements of a higher frequency prevail. intermittent suckling. SHA and FLA oral movements are more intense during continuous suckling.

The distribution of energy in terms of frequency in the oral movements displayed in three dimensions during whole suckling bouts was recorded (Fig. 4). This was the amount of energy used in each frequency band in $30 \mathrm{~s}$ frames during a complete suckling bout, from the time the infant attached to the nipple until spontaneous detachment. The bandwidth of the periodic oral movements displayed during suckling varied in a scale ranging from 0 to $8 \mathrm{~Hz}$ (Fig. 4). The same bandwidth was observed during the whole suckling bout. The pattern of energy used in each one of the frequencies that were displayed by periodical oral movements changed during the suckling bout. The pattern of energy of the SHA movements (Fig. 4a) showed that those of the lowest frequency, that is less than $1 \mathrm{~Hz}$, used higher energy levels during the first few minutes of recording. After the first few minutes, higher energy levels shifted towards oral movements of higher frequencies. The pattern of energy of the FLA movements (Fig. 4b) was similar to that described for the SHA movements, but the energy was 100 times lower. The means \pm SEM of the total amount of power displayed in the distribution of energy in terms of frequency throughout the whole suckling bout was calculated in a group of six babies (Fig. 5). Statistical variation was observed between individual regressions. This variation accounts for $21.4 \%$ of the total variation in the data. However, there was a linear trend of energy that was similar for the whole group $(P<0.001)$. The highest level of energy was displayed during the first 2 min of the suckling bout, after which the energy decreased with fluctuations. The variation observed between babies may be due to asynchronies in the periods of continuous or intermittent suckling activity.

Statistical analysis performed in individual babies showed a correlation between the energy of SHA and FLA waves during a suckling bout $(P<0.001)$. No individual variation was found in slope or in $y$ intercepts between the data for each baby (Fig. 6).

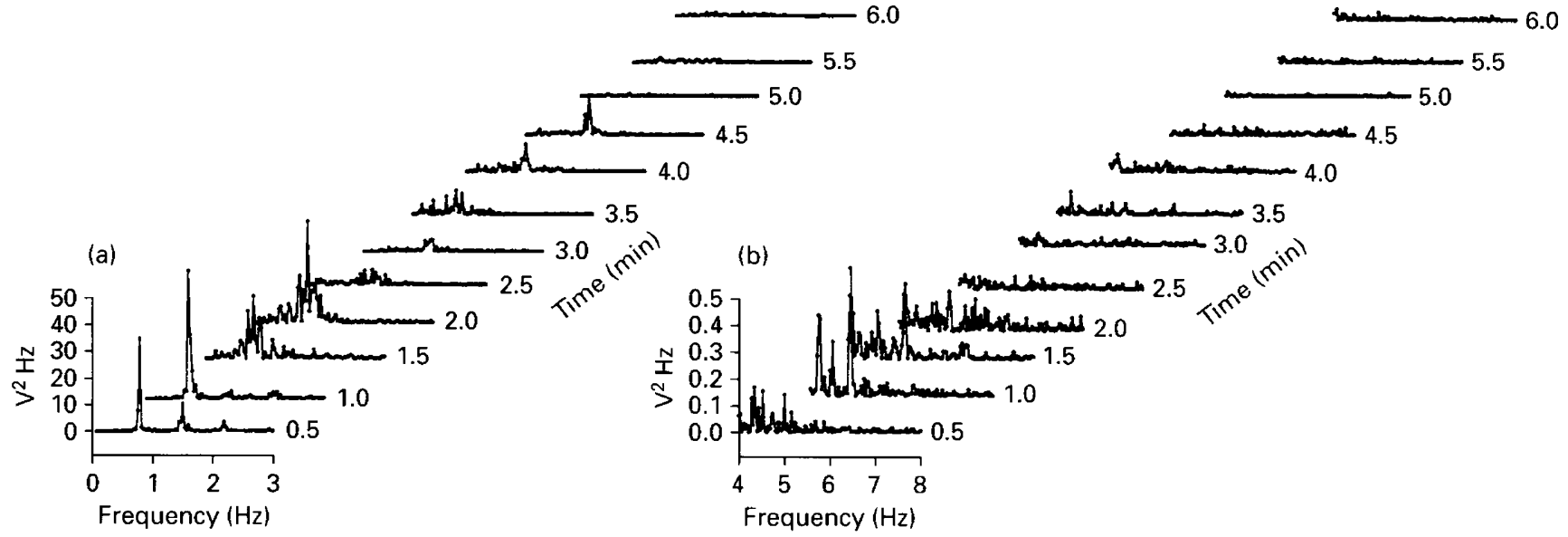

Fig. 4. The distribution of energy in terms of frequency of (a) slow high amplitude (SHA) and (b) fast low amplitude (FLA) oral movements of a breastfeeding human baby during an entire suckling bout in three dimensions. The amount of energy used in each frequency band is shown in frames of $30 \mathrm{~s}$. The energy of SHA and FLA movements changes throughout the suckling bout. The pattern of both movements is similar, but the energy of the FLA movements is 100 times lower. 


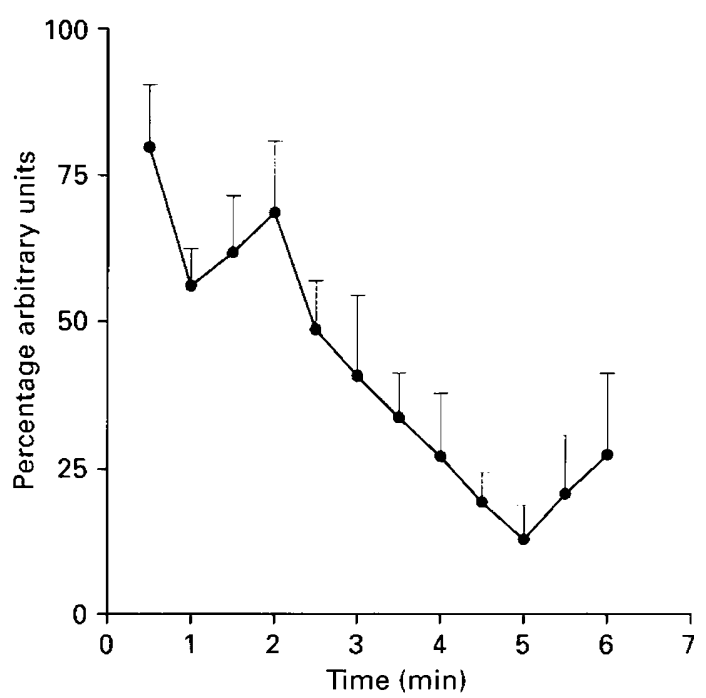

Fig. 5. The total amount of power displayed in the distribution of energy in terms of frequency throughout the whole suckling bout of six breastfeeding human babies. Data are expressed in arbitrary units. Values are means \pm SEM. The presence of linear component trends in the data was checked, accounting for intra- and inter-subject variability. Despite variation, there is a linear trend of energy that is similar for the whole group $(P<0.001)$. The highest level of energy was displayed during the first $2 \mathrm{~min}$, after which the energy decreased.

\section{Discussion}

Oral movements during a suckling bout in babies of 30-60 days of age were recorded using an orokinetogram and were quantified and analysed in the frequency domain after FFT. This is the first study to reveal that the frequency bandwidth of these oral movements is wider $(0-8 \mathrm{~Hz})$ than previously reported by Dreweet and Wooldridge (1979) $(0-3 \mathrm{~Hz})$. This is due to the presence of FLA movements. The same bandwidth of frequencies is observed throughout the whole suckling bout. These data are in agreement with those of Bowen Jones et al. (1982), who reported that the frequency distribution of oral movements is unimodal.

During breastfeeding, babies obtain the milk contained in the lactiferous sinuses (Wooldridge, 1986; Lau et al., 1997). Positive pressure on the nipple from the surface of the tongue is the primary force that evacuates the milk. A roller like peristaltic wave of contraction throughout the surface of the tongue squeezes milk from the ampullae into the oesophagus (Gwynne-Evans, 1951; Ardran et al., 1958; Wooldridge, 1986). Simultaneous recordings from an orokinetogram and a pressure transducer showed that both the SHA and the FLA oral movements recorded by the orokinetogram exert a positive pressure action upon the nipple because they are concurrently detected at the same amplitude by the pressure transducer, which only records positive pressure. The positive pressure at the nipple was considered to represent the component of suckling that corresponds to the squeezing of the nipple (Lau et al., 1997) Therefore, it is possible that the waves recorded by the orokinetogram represent oral activity associated with nipple squeezing. The relationship between SHA movements $(0-3 \mathrm{~Hz})$ and milk ingestion has been studied (Wolff, 1968; Dreweet and Wooldridge, 1979). The FLA movements $(4-8 \mathrm{~Hz})$ described for the first time in this paper are superimposed on the SHA movements. This vibrating type
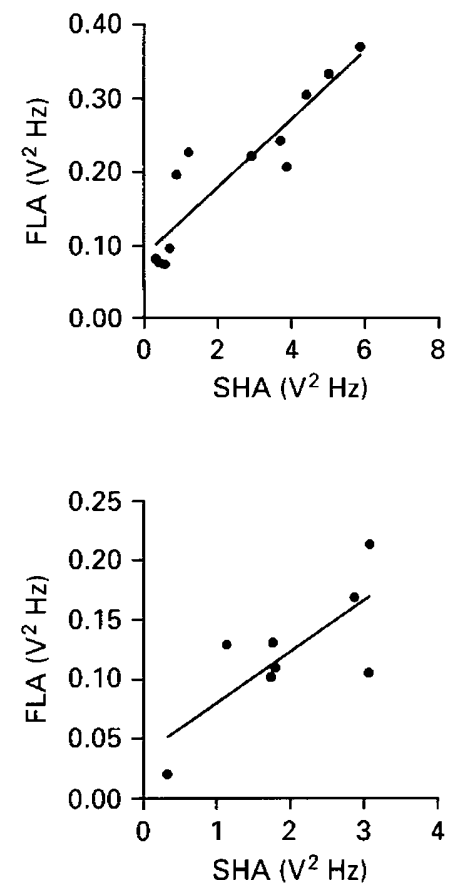
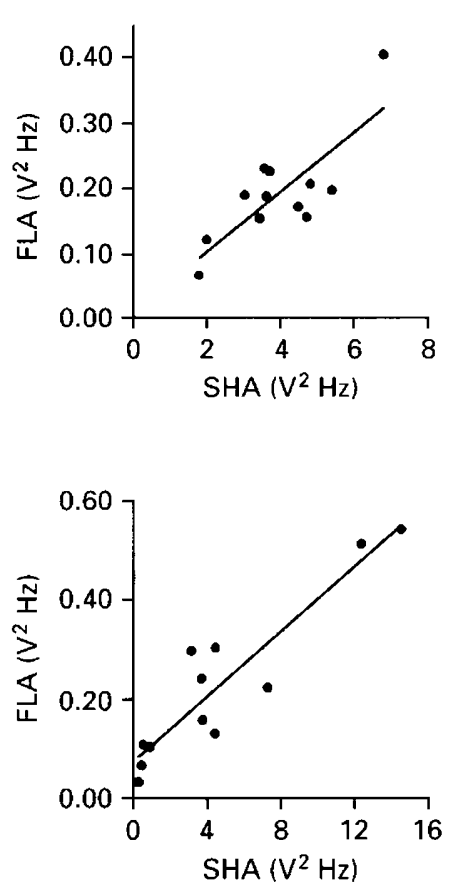
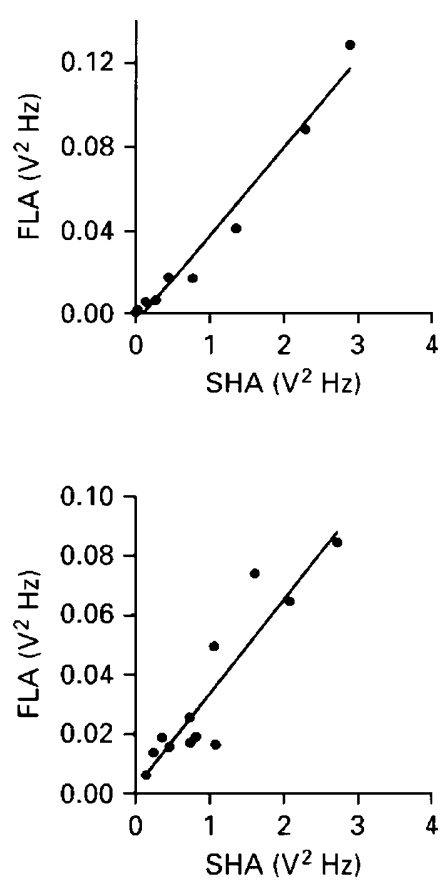

Fig. 6. Correlation $(P<0.001)$ between the energy of slow high amplitude (SHA) and fast low amplitude (FLA) waves throughout a suckling bout in breastfeeding human babies. No individual variation was found in the slope or the $y$ intercepts in the data for each baby. 
of movement may give a tickling stimulus to mechanoreceptors in the nipple and areola due to its high speed and low intensity. Moreover, FLA movements may derive from peristaltic movements of the tongue related to the expression of milk from the nipple. They may propel milk and stimulate mechanoreceptors in the nipple and areola to release hypophysial hormones.

Sampled waveform data may be indistinctly displayed in time or frequency domain. The advantage of displaying the waveforms after FFT is that this algorithm allows storage, quantification and frequency analysis of all the periodical waveforms produced by oral movements throughout the suckling bout, enabling the total oral activity to be measured. The pattern of the distribution of energy in terms of frequency changes in relation with the continuous and intermittent periods of suckling. Throughout the suckling bout, the pattern of SHA movements showed that those of the lowest frequency (under $1 \mathrm{~Hz}$ ) are predominant during the first few minutes of suckling, after which those of higher frequency are most common (about $2 \mathrm{~Hz}$ ). Movements of $1 \mathrm{~Hz}$ correspond to continuous suckling episodes, while those of $2-3 \mathrm{~Hz}$ correspond to intermittent episodes. It has been reported that milk ingestion occurs mainly during episodes of continuous suckling (Wolff, 1968). The pattern of FLA movements observed in the present study showed that these movements are more intense during continuous suckling. The energy displayed by the FLA movements is 100 times lower than the SHA movements. However, the pattern is similar, since the slowest movements of this group are predominantly at the beginning of the suckling bout. Statistical analysis performed in six babies showed that despite variations between individual regressions, there was a similar linear trend of energy in the group. The highest levels of energy were displayed during the first 2 min of the suckling bout and declined thereafter. These results are in accordance with the findings of Prieto et al. (1996), who reported a gradual decrease of suckling activity throughout the suckling bout. This may be explained by the fact that babies obtain the most milk during the first 2 min of suckling, as reported by Lucas et al. (1979). Statistical analysis also showed a correlation between the energy of the SHA and FLA waves throughout the suckling bout. The energy of the SHA and FLA waves increased or decreased simultaneously. If it is assumed that the FLA waves represent peristaltic waves of contraction of the tongue, this type of rapid tongue movement would predominate during continuous suckling episodes in which mainly SHA oral movements occur. Therefore, the peristaltic contraction activity of the tongue would be greater and the stimulation of mechanoreceptors in the nipple caused by this type of rapid movement would be more intense during nutritive suckling episodes.

Oral movements stimulate mechanoreceptors in the nipple which in turn evoke a pattern of nerve action potentials in the mammary nerve (Voloschin et al., 1988). Therefore, it may be assumed that the pattern of the distribution of energy in terms of frequency displayed by oral movements represents the sensorial code used by the infant to induce hormonal changes in the mother. It is important to quantify this stimulus to explore the hormonal reflex action of suckling.

In conclusion, the results obtained in this study show that the Fourier series is useful for measuring the total oral activity during a suckling bout and, consequently, quantifying and statistically analysing the overall magnitude of the suckling stimulus. Use of the Fourier series and the orokinetogram allows novel investigation of the relationship between the overall magnitude of suckling and the reflex release of hormones.

This work was supported by the Consejo Nacional de Investigaciones Científicas y Técnicas de la República Argentina (CONICET); by the Municipalidad de la Ciudad de Buenos Aires (MCBA); and by the Fundación Instituto de Neurobiología. The authors are grateful to Héctor Bianconi, Director of the Obstetric and Gynecology Department, Raúl Ruvinski, Head of the Mother-Infant Department, and Tomás Di Pietro, Director of the Hospital Durand, for their very important help which this work possible. The authors are also very grateful to Diego Voloschin (Musician) and to Pablo Meilan (Bachelor in Physics) for their assistance with the series of Fourier for the study of the oral movements of the babies. The expert statistical assistance of A. Garsd is gratefully acknowledged. The authors express their sincere gratitude to J. H. Tramezzani, M. R. Gallardo and $\mathrm{H}$. Croxatto for revising the manuscript, and M.Golía for assistance with the English version of the manuscript.

\section{References}

Ardran GM, Kemp FH and Lind J (1958) A cineradiographic study of breast feeding British Journal of Radiology 31 156-162

Bowen Jones A, Thompson C and Dreweet RF (1982) Milk flow and suckling rates during breastfeeding Developmental Medicine and Child Netrology 24 $622-633$

Crowder MJ and Hand DJ (1990) Analysis of Repeated Measures Chapman and Hall, New York

Diaz S, Rodriguez G, Marshall G, del Pino G, Casado ME, Miranda P, Schiappacase V and Croxatto HB (1988) Breastfeeding pattern and the duration of lactational amenorrhoea in urban Chilean women Contraception 3837-51

Dreweet RF and Wooldridge M (1979) Suckling pattern of human babies on the breast Early Human Development 314 315-320

Gwynne-Evans E (1951) The organization of the oro-facial muscles in relation to breathing and feeding British Dental Journal 91 135-140

Howie P, McNeilly AS, Houston MJ, Cook A and Boyle H (1982) Fertility after childbirth infant feeding patterns, basal PRL levels and postpartum ovulation Clinical Endocrinology $17315-322$

Lau C, Sheena H, Shulman RJ and Shandler RJ (1997) Oral feeding in low birth weight infants fourtal of Pediatrics 130 561-569

Lucas A, Lucas PJ and Baum JD (1979) Pattern of milk flow in breastfeeding infants The Lancet II 57-58

Prieto CR, Cardenas H, Salvatierra AM, Boza C, Montes CG and Croxatto HB (1996) Suckling pressure and its relationship to milk transfer during breastfeeding in humans Journal of Reproduction and Fertility 108 69-74

Ramirez RW (1985) The FFT: Fundamental and Concepts Prentice Hall Inc., Englewood Cliffs, NJ

Voloschin LM, Décima E and Tramezzani JH (1988) Electronmicroscopic and electrophysiological studies of the teat branch of the XIII thoracic nerve: relationship with lactation in the rat Journal of Endocrinology 118 471-483

Wolff PH (1968) Suckling pattern of infant mammals Brain Behavior and Evolution 1354-367

Wooldridge MW (1986) The anatomy of infant sucking Midwifery 2164-171

Yokoyama Y, Ueda T, Irachara M and Aono T (1994) Release of oxytocin and prolactin during breast massage and suckling in puerperal women European Journal of Obstetrics and Gynecology and Reproductive Biology 53 17-20 